Background: Lupus nephritis (LN) represents a serious manifestation of systemic lupus erythematosus (SLE) which requires timely diagnosis, treatment and monitoring. Kidney biopsy is the gold standard of diagnosis and is instrumental to treatment decisions, however it is not generally performed for monitoring and evaluation of response to treatment. To such purposes, accessible biomarkers, for instance urinary, might be highly advantageous.

Objectives: To evaluate urine-Galectin 3 binding protein (UGAL3BP) as a novel biomarker in biopsy-proven active lupus nephritis (A-LN) in comparison to active non-renal SLE (ANR-SLE), inactive SLE (I-SLE), and in population-based controls $(\mathrm{HC})$. Furthermore, we compared UGAL3BP with known markers of renal pathology including neutrophil gelatinase-associated lipocalin (NGAL), osteopontin (OPN), kidney injury molecule $1(\mathrm{KIM}-1)$, and galectin 3 (GAL3).

Methods: Urine samples from A-LN ( $n=86)$, ANR-SLE $(n=63)$, I-SLE $(n=73)$ and $\mathrm{HC}(\mathrm{n}=48)$ were included. UGAL3BP was measured using a commercial ELISA kit and values, adjusted for u-creatinine levels, were expressed as $\mathrm{ng} / \mathrm{mmol}$. Other markers analyzed according to clinical routine at the Department of Clinical Chemistry at Uppsala University Hospital were also adjusted for u-creatinine levels. Renal biopsies were graded according to the ISN/RPS classification(1) and evaluated for activity and chronicity index. Ten A-LN patients were evaluated before and after induction treatment.

Results: In the A-LN group, median (IQR) levels of u-GAL3BP were 15.8 (6.824.6) $\mathrm{ng} / \mathrm{mmol}$, while in ANR-SLE, I-SLE, HC were significantly lower 4.4 (2.09.0), $2.8(1.7-4.7), 2.0(0.9-4.8)$ respectively (Kruskal-Wallis $p<0.0001)$. Similarly, u-NGAL was found at higher levels in A-LN patients, $3.3(2.0-5.7) \mu \mathrm{g} / \mathrm{mmol}$, with respect to the ANR-SLE 2.0 (0.9-4.5), I-SLE 1.6 (0.8-3.2), and HC 2.4 (1.2-5.3), $(p=0.008)$. The highest levels of OPN were found in the group of I-SLE (190.6 (85.1-299.9) $\mathrm{\mu g} / \mathrm{mmol}$, compared to A-LN 72.98 (37.6-118.1), ANR-SLE 92.3 (58.5-129.7) and HC 76.5 (58.2-120.3), ( $<<0.0001)$. KIM-1 levels differed among groups with higher levels in the A-LN group (188.9 (113.7-309.7) $\mathrm{ng} / \mathrm{mmol})$, in comparison to ANR-SLE 131.4 (92.2-186.1), I-SLE 123.8 (70.3-200.2), and HC 78.2 (68.8-115.1), $(p<0.0001)$. GAL3 showed comparable levels across groups. When exploring the biomarkers across histologic subgroups of LN, U-GAL3BP could discriminate between proliferative and mesangial forms (17.7(9.6-32.5) vs $6.7(5.1-16.1) \mathrm{ng} / \mathrm{mmol}, \mathrm{p}=0.027)$, while it did not discriminate against membranous LN. U-NGAL was higher in proliferative LN 3.7(2.4-5.8) $\mu \mathrm{g} / \mathrm{mmol}$ with respect to membranous $2.4(1.1-3.8)(p=0.01)$, while mesangial LN showed comparable levels. OPN, KIM-1 and GAL3 were comparable across groups.

In the ten patients with available samples after induction therapy (mycophenolate mofetil (MMF) in 4, rituximab (RTX) in one, cyclophosphamide in 5 (one combined with MMF and one with RTX), u-GAL3BP showed a significant decrease of median levels from 218.8 to $41.5 \mathrm{ng} / \mathrm{mmol}$ (Wilcoxon $\mathrm{p}=0.03$ ). $\mathrm{u}$-GAL3BP associated with renal activity in class III/IV LN $(R=0.42, p=0.004)$.

Conclusion: Among the tested markers, high uGal3BP adjusted for creatinine was found to be a promising marker of renal involvement in SLE patients and associated with renal activity in patients with proliferative forms (class III/IV) of LN. A decrease was further seen following therapy, suggesting that $u$ GAL3-BP could be used to monitor renal activity.

REFERENCES:

[1] Weening JJ, D’Agati VD, Schwartz MM, Seshan SV, Alpers CE, Appel GB, et al. The classification of glomerulonephritis in systemic lupus erythematosus revisited. J Am Soc Nephrol. 2004;15(2):241-50.

[2] Houssiau FA, Vasconcelos C, D'Cruz D, Sebastiani GD, Garrido Ed Ede R, Danieli MG, et al. Immunosuppressive therapy in lupus nephritis: the Euro-Lupus Nephritis Trial, a randomized trial of low-dose versus high-dose intravenous cyclophosphamide. Arthritis Rheum. 2002;46(8):2121-31.

Disclosure of Interests: Francesca Faustini Speakers bureau: I have received speaking fees, last time more than two years ago, Helena Idborg: None declared, Elisabet Svenungsson: None declared, Sven Poetzsch Employee of: Merck Serono, Shinji Okitsu Employee of: Merck Serono, Anders Larsson: None declared, Iva Gunnarsson: None declared

DOI: 10.1136/annrheumdis-2021-eular.3477

\section{POS0185 IDENTIFICATION AND VALIDATION OF TWO NOVEL SERUM BIOMARKERS ASSOCIATED WITH THE SEROLOGICAL STATUS OF RHEUMATOID ARTHRITIS PATIENTS.}

V. Calamia ${ }^{1}$, L. Lourido ${ }^{1}$, P. Fernández Puente ${ }^{1,2}$, A. Illiano ${ }^{3}$, R. Paz González ${ }^{1}$, B. Rocha Loureda ${ }^{1}$, L. Collado Rodríguez ${ }^{1}$, E. Perez-Pampín ${ }^{4}$, C. RuizRomero ${ }^{1}$, A. Gonzalez ${ }^{4}$, F. J. Blanco' ${ }^{1}{ }^{1}$ Instituto de Investigación Biomédica de A Coruña (INIBIC), Complexo Hospitalario Universitario de A Coruña (CHUAC), Sergas, Universidade da Coruña (UDC), Unidad de Proteómica, Grupo de Investigación de Reumatología (GIR), A Coruña, Spain; ${ }^{2}$ Universidad de A Coruña (UDC), Centro de Investigaciones Científicas Avanzadas (CICA), A Coruña, Spain; ${ }^{3}$ University of Naples Federico II, Department of Chemical Sciences, Naples, Italy; ${ }^{4}$ Instituto de Investigación Sanitaria (IDIS). Hospital Clínico Universitario de Santiago (CHUS), Experimental and Observational Rheumatology and Rheumatology Unit, Santiago de Compostela, Spain
Background: Despite the diagnostic value of Rheumatoid Factor (RF) and Anti-Citrullinated Protein Antibodies (ACPA), more serological markers are needed in order to improve early diagnosis and treatment response of the Rheumatoid Arthritis (RA) patients. Increased knowledge about how these two major autoreactivities arise is crucial for understanding how RA develops and what mechanisms drive pathogenesis.

Objectives: We aimed to investigate, using a proteomic strategy, novel protein biomarkers associated with RF and/or ACPA that might be useful to stratify seropositive and seronegative RA patients.

Methods: A shotgun proteomic analysis was performed on 80 sera from the RA cohort of the Rheumatology Unit of the University Hospital of Santiago de Compostela (CHUS). Sera were classified as seropositive or seronegative according to their RF and ACPA values, and were then analyzed employing the iTRAQ labelling technique (Sciex) followed by LC-MALDI-MS/MS analysis (MALDI-TOF). A Multiple Reaction Monitoring (MRM) method was subsequently developed using the Skyline Software for the simultaneous quantification of 26 peptides belonging to ten putative protein biomarkers. The quantitative targeted analysis was performed using peptides with isotope labelled amino acids as internal standards Serum levels of orosomucoid 1 (ORM1) and haptoglobin (HPT) were measured using commercially available ELISA Kits in the whole RA cohort $(n=260)$ from the Rheumatology Unit of the University Hospital of A Coruña (HUAC).

Results: For the initial screening, eighty sera were grouped according to the ACPA RF status in 4 pools (20 patients/pool). Using an iTRAQ technology-based quantitative proteomic approach, the abundance of eleven proteins was altered in the sera from $A C P A^{\text {pos }} / R^{\text {pos }}, 13$ proteins in $A C P A^{\text {neg }} / R^{\text {pos }}$ and 12 proteins in ACPApos/RF ${ }^{\text {neg }}$, compared to $A C P A^{\text {neg }} / R^{\text {neg }}$. Vitamin $D$ binding protein (VTDB) was the unique protein that resulted increased in all the comparisons. For the biomarker verification phase, all the samples from the CHUS cohort were analyzed individually $(n=80)$. Using the MRM technology, 26 peptides belonging to ten putative protein biomarkers associated with double positivity were simultaneously quantified. The statistical analysis showed a significant modulation of 9 peptides (belonging to 4 different proteins) in $\mathrm{ACPA}^{\mathrm{pos}} / \mathrm{RF}^{\mathrm{pos}}, 7$ peptides (5 proteins) in $\mathrm{ACPA}^{\mathrm{neg}} / \mathrm{RF}^{\mathrm{pos}}$, and 9 peptides (6 proteins) in $A C P A^{\text {pos }} / R^{\text {neg }}$ compared to $A C P A^{\text {neg }} / R^{\text {neg }}(p<0.05)$. Two acute phase reactants (ORM1 and HPT) displayed the same modulation in both screening and verification phases, thus confirming their association with the double positivity. Finally, in the biomarker validation phase, a total of 260 patients from CHUAC were included (Table 1). RA patients were classified as follows: (1) 112 patients $(43.1 \%)$ were $\mathrm{ACPA}^{\mathrm{pos}} / \mathrm{RF}^{\mathrm{pos}}$; (2) 73 patients $(28.1 \%)$ were $\mathrm{ACPA}^{\text {neg }} / \mathrm{RF}^{\text {neg }}$ (3) 51 patients (19.6\%) were $\mathrm{ACPA}^{\text {neg }} / \mathrm{RF}^{\text {pos }}$; and (4) 24 patients $(9.2 \%)$ were ACPA${ }^{\mathrm{pos}} / \mathrm{RF}^{\mathrm{neg}}$. Serum levels of ORM1 and HPT (Figure 1), measured by commercial immunoassays, confirmed their increased values in double seropositive patients $(p=0,0053$ ORM1; $p=0,0026$ HPT). Finally, the increased level of ORM1 resulted associated with RF rather than ACPA status $\left(p=0,0008 A C P A^{\text {neg }} / R^{p 0 s}\right)$; whereas HPT was associated with ACPA rather than $R F$ status $\left(p=0,0112 A^{A C P A}{ }^{p o s} / R F^{\text {neg }}\right.$ ).

Table 1. The different phases of RA biomarker development followed in this study.

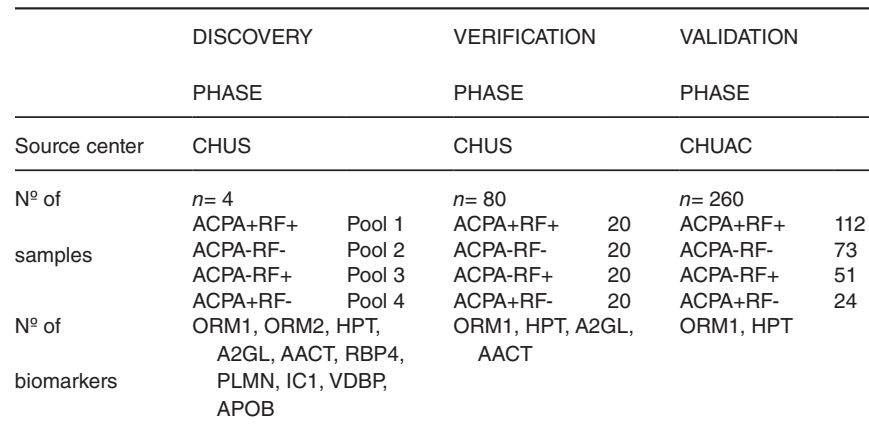
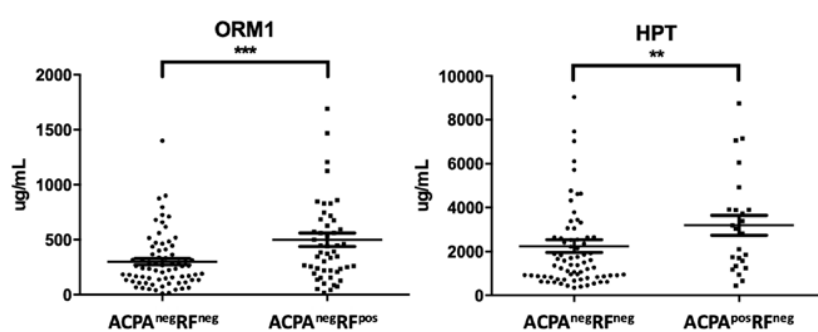

Figure 1: ORM1 levels are increased in RA patients seropositive for RF $(499 \pm 437 \mathrm{ug} / \mathrm{mL}$ in $A_{C P A^{\text {neg }}} R^{\text {pos }}$ vs $300 \pm 248 \mathrm{ug} / \mathrm{mL}^{\text {in }}$ ACPA $\left.{ }^{\text {reg RF }} \mathrm{F}^{\text {neg }}\right)$. HPT levels are statistically significant increase in RA patients seropositive for ACPA (3193+2233 ug/mL in ACPA ${ }^{\text {pos RF }}{ }^{\text {neg }} \mathrm{vs} 2046 \pm 1795 \mathrm{ug} / \mathrm{mL}$ in ACPA $^{\text {neg RF }}{ }^{\text {neg }}$.

Figure 1. 
Conclusion: The determination of ORM1 and HPT in sera provides novel information useful for patient stratification, which might improve diagnostic and prognostic approaches and facilitate the development of personalized medicine strategies in RA.

Acknowledgements: This work is supported by grants from Fondo de Investigación Sanitaria (RD16/0012/0002, PT17/0019/0014) integrated in the National Plan for Scientific Program, Development and Technological Innovation 20132016 and funded by the ISCIII-General Subdirection of Assessment and Promotion of Research-European Regional Development Fund (FEDER) "A way of making Europe".

Disclosure of Interests: None declared

DOI: 10.1136/annrheumdis-2021-eular.2533

\section{POS0186 \\ METABOLOMIC SIGNATURES FOR KNEE CARTILAGE VOLUME LOSS OVER 10 YEARS}

Z. Xie $^{1,2}$, D. Aitken ${ }^{3}$, M. Liu' ${ }^{1}$, G. Lei ${ }^{2}$, G. Jones ${ }^{3}$, G. Zhai ${ }^{1}{ }^{1}$ Memorial University of Newfoundland, Division of Biomedical Sciences (Genetics), Faculty of Medicine, St. John's, Canada; ${ }^{2}$ Central South University, Xiangya Hospital, Changsha, China; ${ }^{3}$ University of Tasmania, Menzies Institute for Medical Research, Hobart, Australia

Background: Osteoarthritis $(\mathrm{OA})$ is the most common form of arthritis, and its impact is increasing year by year due to an aging population and lack of effective treatments. One of the main structural pathological changes of $O A$ is the loss of articular cartilage. Tools that can predict cartilage loss would help identify people at high risk, thus preventing OA development.

Objectives: Using a metabolomics approach, the current study aimed to identify serum metabolomic signatures for predicting the loss of knee cartilage volume over 10 years in a well-established community-based cohort - the Tasmania Older Adult Cohort (TASOAC).

Methods: TASOAC is an on-going, prospective, population-based study of older adults who were randomly selected from the roll of electors in Southern Tasmania, Australia. Participants had a right knee magnetic resonance imaging (MRI) scan at baseline and a 10-year follow-up. Cartilage volume was measured in the medial, lateral, and patellar compartments and change in cartilage volume over 10 years was calculated as percentage change per year. Fasting serum samples collected at 2.6-year follow-up were metabolomically profiled using the TMIC Prime Metabolomics Profiling Assay which measures a total of 143 metabolites. 129 metabolite concentrations passed the quality control and the pairwise ratios of them as the proxies of enzymatic reaction were calculated. Linear regression models were used to test the association between each of the metabolite ratios and change in cartilage volume in each of the knee compartments with adjustment for age, sex, and body mass index (BMI). The significance was defined at $a=3.0 \times 10^{-6}$ to control multiple testing of 16,512 ratios with Bonferroni method.

Results: A total of 344 participants ( $51 \%$ females) were included. The mean baseline age was $62.83 \pm 6.13$ years and the mean BMI was $27.48 \pm 4.41 \mathrm{~kg} / \mathrm{m}^{2}$. The average follow-up time was $10.84 \pm 0.66$ years. Cartilage volume reduced by $1.34 \pm 0.72 \%, 1.06 \pm 0.58 \%$, and $0.98 \pm 0.46 \%$ per year in the medial, lateral, and patellar compartments, respectively. Our data showed that an increased ratio of hexadecenoylcarnitine (C16:1) to tetradecanoylcarnitine $(\mathrm{C} 14)$ was associated with a $0.12 \pm 0.02 \%$ per year reduction in patellar cartilage volume $\left(p=8.80 \times 10^{-7}\right)$. An increased ratio of hexadecenoylcarnitine (C16:1) to dodecanoylcarnitine (C12) was also associated with a $0.12 \pm 0.02 \%$ per year reduction in patellar cartilage volume $\left(p=2.66 \times 10^{-6}\right)$. While there were several metabolite ratios associated with cartilage volume loss in the medial and lateral compartments, none of them reached the predefined significance level.

Conclusion: Our data suggested that alteration of fatty acid $\beta$-oxidation is involved in knee cartilage loss, especially in the patellar compartment, and the serum ratio of $\mathrm{C} 16: 1$ to $\mathrm{C} 14$ and to $\mathrm{C} 12$ could be used to predict long-term patellar cartilage loss.

Acknowledgements: We thank all the study participants who made the study possible. The original TASOAC study was supported by the National Health and Medical Research Council (NHMRC) and the current study was supported by the Canadian Institutes of Health Research (ClHR).

Disclosure of Interests: None declared

DOI: 10.1136/annrheumdis-2021-eular.2735

\section{POS0187 THE PROGNOSTIC FACTORS IN PREDICTING RELAPSE FOR IGG4-RELATED DISEASE: A LONG-TERM STUDY BASED ON THE CLINICAL AND PATHOLOGICAL CHARACTERISTICS OF THE PATIENTS}

Z. Ji ${ }^{1}$, L. Chen ${ }^{2}$, L. Ma ${ }^{1}$, L. Zhang ${ }^{1}$, H. Chen ${ }^{1}$, L. Ma ${ }^{1}$, L. Jiang ${ }^{1} .{ }^{1}$ Shanghai Zhongshan hospital, Fudan University, Rheumatology, Shanghai, China; ${ }^{2}$ Shanghai Zhongshan hospital, Fudan University, Pathology, Shanghai, China
Background: The relationship between the pathological findings and disease relapse has not been well established.

Objectives: We aim to investigate the clinical and pathological manifestations in relation with disease relapse in IgG4-RD, as well as identify prognostic factors in predicting relapsed disease.

Methods: This study enrolled 71 patients newly diagnosed with IgG4-RD between Jan 2011 and April 2020, all of whom had received pathological examinations. Their pathological manifestations and clinical data were collected. Multivariate Cox regression and AUC (area under curve) were used to identify predictors for relapsed disease and assess the predictive value of these predictors, respectively. Results: During a follow-up period of 26 (range, 6-123) months, 4.2\% (3/71) patients died. The remaining 68 patients were all treated with glucocorticoids with or without immunosuppressor continuously. By the end of follow-up, 47 (69.1\%) patients sustained clinical remission, and 21 (30.9\%) patients suffered relapsed disease with a median relapse time at 10 (6-30) months. We found that IgG4 $\geq$ 6.5g/L (OR 1.52-11.06), IgG $\geq 20.8 \mathrm{~g} / \mathrm{L}$ (OR 1.11-7.23), IgG4-RD responder index $(\mathrm{RI}) \geq 9$ (OR 1.28-11.37), and more IgG4 ${ }^{+}$plasma cell infiltration ( $\geq 60 / \mathrm{HPF}$ in visceral organs, or $\geq 200$ / HPF in head and neck organs) (OR 1.79-22.41) were all independent predictive factors for disease relapse. A prognostic score was explored for predicting recurrence in IgG4-RD, including three predictive factors (IgG $\geq 20.8 \mathrm{~g} / \mathrm{L}, \lg \mathrm{G} 4-\mathrm{RD} \mathrm{RI} \geq 9$, and more $\operatorname{lgG} 4^{+}$plasma cell infiltration). The three-year relapse rate for the patients with no, one, two, and three risk factors were $0 \%, 27.3 \%, 66.7 \%$, and $100 \%$, respectively.

Conclusion: Patients' earlier lgG4 $\geq 6.5 \mathrm{~g} / \mathrm{L}, \mathrm{IgG} \geq 20.8 \mathrm{~g} / \mathrm{L}$, IgG4-RD RI $\geq 9$, and more $\operatorname{lgG} 4^{+}$plasma cell infiltration independently predicted disease relapse. We explored a prognostic score for predicting recurrence in IgG4-RD include three predictive factors ( $\operatorname{lgG} \geq 20.8 \mathrm{~g} / \mathrm{L}$, IgG4-RD $\mathrm{RI} \geq 9$, and more $\operatorname{lgG} 4^{+}$plasma cell infiltration), which might be used to evaluate the risk of recurrence in IgG4-RD.

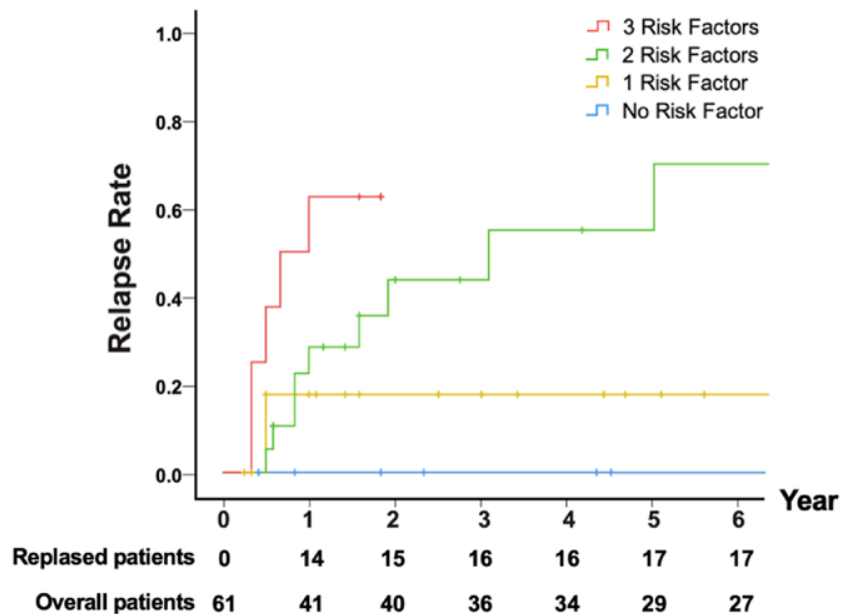

\section{REFERENCES:}

[1] DESHPANDE V, ZEN Y, CHAN J K, et al. Consensus statement on the pathology of IgG4-related disease[J]. Mod Pathol,2012,25(9): 1181-1192.

[2] JENNETTE J C, FALK R J, BACON P A, et al. 2012 revised International Chapel Hill Consensus Conference Nomenclature of Vasculitides[J]. Arthritis Rheum,2013,65(1): 1-11.

[3] OKAZAKI K, UMEHARA H. Are Classification Criteria for IgG4-RD Now Pos sible? The Concept of IgG4-Related Disease and Proposal of Comprehensive Diagnostic Criteria in Japan[J]. Int J Rheumatol,2012,2012: 357071.

[4] SHIMOSEGAWA T, CHARI S T, FRULLONI L, et al. International consensus diagnostic criteria for autoimmune pancreatitis: guidelines of the International Association of Pancreatology[J]. Pancreas,2011,40(3): 352-358.

[5] OHARA H, OKAZAKI K, TSUBOUCHI H, et al. Clinical diagnostic criteria of IgG4-related sclerosing cholangitis 2012[J]. J Hepatobiliary Pancreat Sci,2012,19(5): 536-542.

[6] KAWANO M, SAEKI T, NAKASHIMA H, et al. Proposal for diagnostic criteria for IgG4-related kidney disease[J]. Clin Exp Nephrol,2011,15(5): 615-626.

[7] GOTO H, TAKAHIRA M, AZUMI A. Diagnostic criteria for IgG4-related ophthalmic disease[J]. Jpn J Ophthalmol,2015,59(1): 1-7.

[8] MATSUI S, YAMAMOTO H, MINAMOTO S, et al. Proposed diagnostic criteria for IgG4-related respiratory disease[J]. Respir Investig,2016,54(2): 130-132.

[9] WEN ZHANG J H S. Management of IgG4-related disease[J]. Lancet Rheumatol,2019,1: e55-e65.

[10] EBBO M, DANIEL L, PAVIC M, et al. IgG4-related systemic disease: features and treatment response in a French cohort: results of a multicenter regis try[J]. Medicine (Baltimore),2012,91(1): 49-56.

Disclosure of Interests: None declared

DOI: 10.1136/annrheumdis-2021-eular.3391 